\title{
Influence of nanostructure on the device performance and charge recombination dynamics of P3HT:PCBM solar cells
}

\author{
LI Dan, XING YaDong, YUE He, ZHANG JianPing \& AI XiCheng* \\ Department of Chemistry, Renmin University of China, Beijing 100872, China
}

Received March 6, 2012; accepted March 25, 2012; published online June 14, 2012

In this paper, we investigated the recombination dynamics of photogenerated charge carriers in a poly(3-hexylthiophene) (P3HT):[6,6]-phenyl- $\mathrm{C}_{61}$-butyric acid methyl ester $\left(\mathrm{PC}_{61} \mathrm{BM}\right)$ blend system with donor-acceptor ratio of 1:1 before and after solvent annealing treatment. The technique of transient photocurrent and photovoltage measurements were used, and charge carriers were photogenerated by a $7 \mathrm{~ns}$ laser pulse at room temperature $(298 \mathrm{~K})$. In transient photocurrent measurement, we observed some differences in the saturation extracted charge in P3HT:PCBM solar cells with different power efficiencies. In addition, the bimolecular recombination coefficient $\beta$ is found to be $3.5 \times 10^{-13} \mathrm{~cm}^{3} \mathrm{~s}^{-1}$ for annealed devices, while $9.5 \times 10^{-12} \mathrm{~cm}^{3} \mathrm{~s}^{-1}$ for as-cast devices. In the transient photovoltage measurement, we found that the photovoltage decay can be fitted by power-law equation at long time scale. The exponent parameter $\alpha$ is 2.6 for annealed devices, which can be described as trap-free bimolecular recombination; $\alpha$ is 1.76 for as-cast device due to the trap-limited bimolecular recombination. These experimental results indicate that the nanomorphology of active layer indeed have influence on charge carriers dynamics in P3HT:PCBM blend systems.

poly(3-hexylthiophene), nanoscale morphology, bimolecular recombination, transient photocurrent and photovoltage

Citation: $\quad$ Li D, Xing Y D, Yue H, et al. Influence of nanostructure on the device performance and charge recombination dynamics of P3HT:PCBM solar cells. Chin Sci Bull, 2012, 57: 3436-3441, doi: 10.1007/s11434-012-5204-1

Bulk heterojunction (BHJ) organic photovoltaics (OPV) are potential candidates as low-cost, low weight alternative energy sources to convert the energy of sunlight into electricity. They also have achieved remarkable power conversion efficiency (PCE) approaching 10\% [1]. The general power conversion processes include light absorption and exciton generation, exciton diffusion, exciton dissociation and charge generation, charge transport and collection. All steps occur in the photoactive layer of the device and are governed by the photoactive layer [2]. The photoactive layer needs to be about 100-200 nm thick in order to absorb more incident light, to have a $10 \mathrm{~nm}$ diffusion length for an exciton [3,4], a large donor/acceptor interfacial area for efficient exciton dissociation, and a continuous pathways for charge carrier transport etc. Ideal nanostructure of the photoactive layer is considered to be an interpenetrating network of donor and acceptor. The performances of devices are determined by the crystallinity

\footnotetext{
*Corresponding author (email: xcai@ ruc.edu.cn)
}

degree of the photoactive layer on the nanoscale. Therefore, the photoactive layer is a key factor for further improving the efficiency of the BHJ organic photovoltaics.

It is well-known that poly(3-hexylthiophene-2,5-diyl) (P3HT):[6,6]-phenyl- $\mathrm{C}_{61}$-butyric acid methyl ester $\left(\mathrm{PC}_{61} \mathrm{BM}\right)$ blend system is usually used as a model to investigate the relationship between the nanostructure of the photoactive layer and the dynamics of charge carriers. The P3HT: PCBM solar cells can easily be processed from solution. In this solution process, the nanostructure of the organic solar cells can be modified by different processing conditions, solvent choice to dissolve the donor and acceptor $[5,6]$, weight ratios of donor and acceptor [7], post-annealing treatments $[8,9]$ etc. A series of microscopic techniques are used to analyze the nanoscale morphology, such as atomic force microscopy (AFM) [10,11], conducting AFM [12,13], electrostatic force microscopy (EFM) $[14,15]$ and scanning Kelvin probe microscopy (SKPM) [14,16]. Additionally, several experimental techniques have been used to probe the 
charge carrier transport and loss mechanism in polymer/ fullerene solar cells, such as charge extraction by linear increasing voltage (CELIV) [17-19], transient absorption spectroscopy (TAS) [19,20], monitoring of transient photocurrent and photovoltage and charge extraction [21,22], and femosecond transient absorption spectroscopy [23,24]. However, it is still under discussion how the nanostructure limits the physical processes, and accordingly influences the power conversion efficiency of the solar cells.

In this study, we investigated the relationship between the nanostructure and charge carrier recombination dynamics, and try to understand how the nanostructure limits the performance of P3HT:PCBM solar cells using transient photocurrent and transient photovoltage measurements. We investigated the nanostructure of P3HT:PCBM using UV-vis absorption spectroscopy, atomic force microscopy (AFM) and X-ray diffraction, while transient photocurrent and photovoltage measurements were used to probe the dynamics of charge carrier in the solar cells.

\section{Materials and methods}

\subsection{Materials}

Regioregular poly(3-hexylthiophene) (RR-P3HT, head-totail $>90 \%, M_{\mathrm{w}}=43462, M_{\mathrm{w}} / M_{\mathrm{n}}=2.9 . M_{\mathrm{w}}$ : weight-average molecular weight, $M_{\mathrm{n}}$ : number-average molecular weight) and PCBM ( 99\%) were both used as received from Sigma-Aldrich without further purified. P3HT and PCBM were mixed together at $1: 1$ weight ratio into the $o$ dichlorobenzene $(o-\mathrm{DCB})$, the concentration of the blend solution was $20 \mathrm{mg} \mathrm{mL}^{-1}$ and stirred overnight. ITO coated glass was treated by ultrasonic treatment in detergent, and cleaned with deionized water, acetone, ethanol and iso-propyl alcohol successively. After baking at $120^{\circ} \mathrm{C}$ for $20 \mathrm{~min}$ in an oven, the poly(3,4-ethylenedioxythiophene): poly(styrenesulfonate) (PEDOT:PSS) layer ( 40 nm) was spin-coated onto a precleaned ITO coated glass, and subsequently annealed at $100^{\circ} \mathrm{C}$ for $10 \mathrm{~min}$. The P3HT:PCBM photoactive layer was spin-coated from the blend $o$ dichlorobenzene solution at a speed of $1000 \mathrm{r} \mathrm{min}^{-1}$ for $30 \mathrm{~s}$. For the as-cast films, they just were left in the argon glovebox $\left(\mathrm{O}_{2}\right.$ and $\mathrm{H}_{2} \mathrm{O}$ concentration both below $\left.10^{-7}\right)$. Annealing treatment was performed by leaving the P3HT:PCBM blend films on a petri dish under $o$-DCB vapour atmosphere in the argon glovebox. Some films used as optical and morphology characterization were prepared by the same procedure before the aluminum cathode deposition. Others had thermally evaporated an aluminum electrode with a thickness of $\sim 80$ nm under a pressure of less than $10^{-3} \mathrm{~Pa}$. The structure of the solar cell is ITO/PEDOT:PSS /P3HT:PCBM/Al.

\subsection{Methods}

Steady absorption spectrum was measured on a Cray-50
(Varian, USA) absorption spectrometer. Atomic force microscopy (AFM) (D3100, Vecco) instrument was used to measure the morphology of the as-cast and annealed films on a tapping mode. X-ray diffraction spectrometer (XRD7000, Shimadzu) was used to examine the intermolecular ordering of the blend films. The X-ray beam was generated by copper $(\mathrm{K} \alpha)$ target, using a tube of voltage of $40 \mathrm{kV}$ at electron beam current of $30 \mathrm{~mA}$. Current-voltage $(I-V)$ curves were performed using Keithley 4200 source meter unit under an AM 1.5 G illumination at $100 \mathrm{MW} \mathrm{cm}^{-2}$, provided by the Thermo Oriel solar simulator.

For the transient photocurrent and photovoltage measurements, the devices were placed in a holder and illuminated from the ITO side by a 7 ns pulse of Nd:YAG laser (Quanta-Ray PRO-170, Spectra Physics) at $532 \mathrm{~nm}$ excitation wavelength. The laser system was running at a repetition rate of $2.177 \mathrm{~Hz}$. The light intensity on the sample varies under a series of optical density filters. The transient photocurrent and photovoltage data were measured from the voltage drop across $50 \Omega$ and $1 \mathrm{M} \Omega$ input terminal of an oscilloscope (Lecroy WaveSurfer 64XS, $600 \mathrm{MHz}$ ), respectively. In order to improve the signal-to-noise ratio, the transient photocurrent and photovoltage data were obtained by an average of up to 100 measurements. All measurements were carried out at room temperature (298 K).

\section{Results and discussion}

\subsection{Current-voltage curves}

The influence of annealing treatment on device performance is shown in Figure 1 and photovoltaic parameters are also summarized inset. The highest power efficiency is $1.97 \%$ (straight line), which is obtained from the devices annealed under $o$-DCB solvent vapour environment. In order to find the exact reason for the different performances between annealed and as-cast devices, we used several steady and

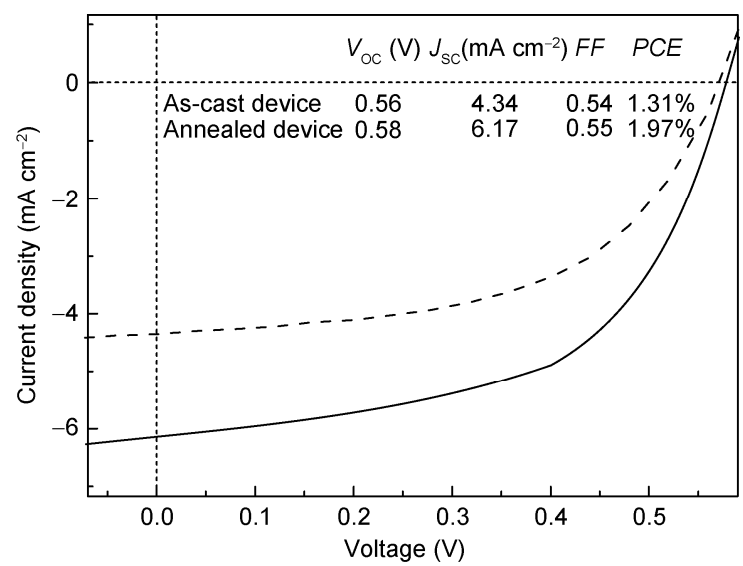

Figure 1 Current-voltage ( $I-V)$ characteristics of P3HT:PCBM solar cells

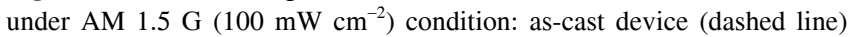
and annealed device (straight line). The inset is the photovoltaic performance parameters of the solar cells. 
transient dynamics measurements to investigate the relationship between nanostructure and charge recombination dynamics.

\subsection{UV-vis absorption spectra and XRD data}

UV-vis and XRD spectrums were examined to investigate the effect of the solvent annealing on the optical and structural properties of the P3HT:PCBM films. Figure 2 shows the UV-vis absorption spectra of P3HT:PCBM blend films before (dash line) and after (straight line) solvent annealing. The P3HT:PCBM film exhibits an absorption peak at 516 $\mathrm{nm}$, and two shoulders at 550 and $600 \mathrm{~nm}$, respectively. The two shoulders are both responsible for the crystallinity of the P3HT:PCBM films [25-28]. Especially the prominent peak at $\sim 600 \mathrm{~nm}$ is attributed to the interchain interactions and $\pi$-stacking structure of P3HT films. After annealing the film in an $o$-DCB solvent vapor environment, there is a pronounced red-shift in the region of P3HT absorption, which means annealing does increase the crystallinity of P3HT chains and results in a highly ordered structure of P3HT:PCBM blend films.

Figure 3 presents the X-ray diffraction (XRD) data of P3HT:PCBM film before and after solvent annealing. The maximum diffraction peak at $5.44^{\circ}$ in blend films shows the separation distance $(d=16.4 \AA)$ in the hexyl side chain orientation, (100) lattice plane. For the annealed blend films, there are two other obvious peaks at $11^{\circ}$ and $16^{\circ}$, which mean the reflections of (002) and (003) lattice planes, respectively. The above results indicate that the crystallinity of P3HT:PCBM blend films can be increased within the phase-separated networks by solvent annealing treatment [29].

\subsection{AFM images}

We investigated the effects of solvent annealing on morphology of the P3HT:PCBM films by the AFM instrument,

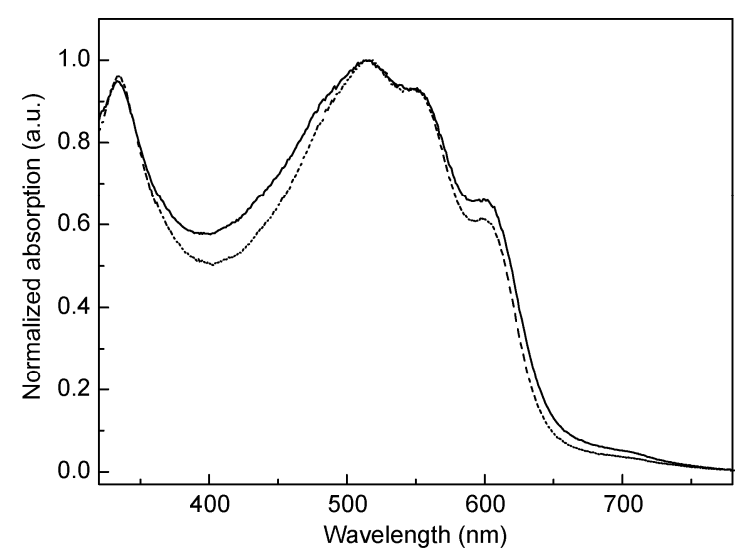

Figure 2 UV-vis absorption spectra of P3HT:PCBM blend films before (dashed line) and after solvent annealing (solid line). The spectra are normalized at $516 \mathrm{~nm}$.

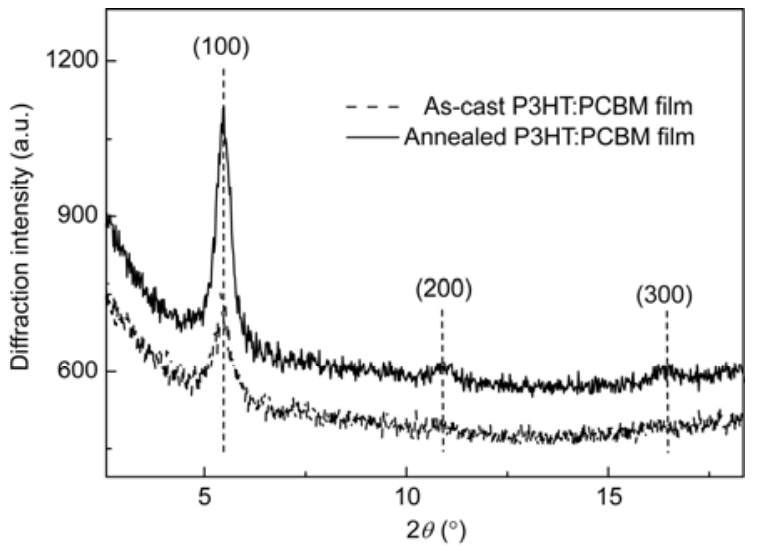

Figure 3 X-ray diffraction data of P3HT:PCBM blend films before (dashed line) and after (solid line) solvent annealing treatment.

as displayed in Figure 4. The height and phase images of pristine P3HT film (Figure 4(a) and (d)) clearly reveal the ordered P3HT polymer fibrils. But its nanostructure was disturbed by adding PCBM molecules just as that have been shown in Figure 4(b) and (e). The as-cast blend films are homogeneous morphology without clear phase separation. After solvent annealing treatments, the blend film has enough time for self-organization to recover ordered structures (Figure 4(c) and (f)). Additionally, the AFM and XRD results provide evidence that the highly crystallinity and good phase-separation of P3HT:PCBM solar cells can be produced by the solvent-annealing treatment, which typically corresponds to excellent device performance $[8,30]$.

\subsection{Transient photocurrent and photovoltage measurements}

In order to investigate the macroscopic charge transport and recombination dynamics in the two devices (before and after annealing treatment), we performed the transient photocurrent and transient photovoltage measurements at room temperature. Figure 5 (a) shows the transient photocurrent data at different light intensities. With increasing light intensity, the photogenerated charge carriers are also increasing which corresponds to the extraction current. On the other hand, extracted charges saturate at the end of light pulse laser and become independent of the intensity for high light intensity (Figure 5(b)), which attributes to bimolecular recombination. As the light intensity increases, more photogenerated charge carrier is in the bulk of the solar cell with a larger possibility of the charge recombination. The extracted charge $Q_{e}$ is inherent to the nanomorophology of the active layer of these P3HT:PCBM solar cells, the nanomorphology with good phase-separation and interpenetrating networks has more pathways for charge transport and be collected at the relevant electrodes, which will results in higher extracted charge $Q_{e}$, and accordingly relate to its power conversion efficiency. Therefore, we can get the bimolecular recombination parameter $\beta$ from the expression 

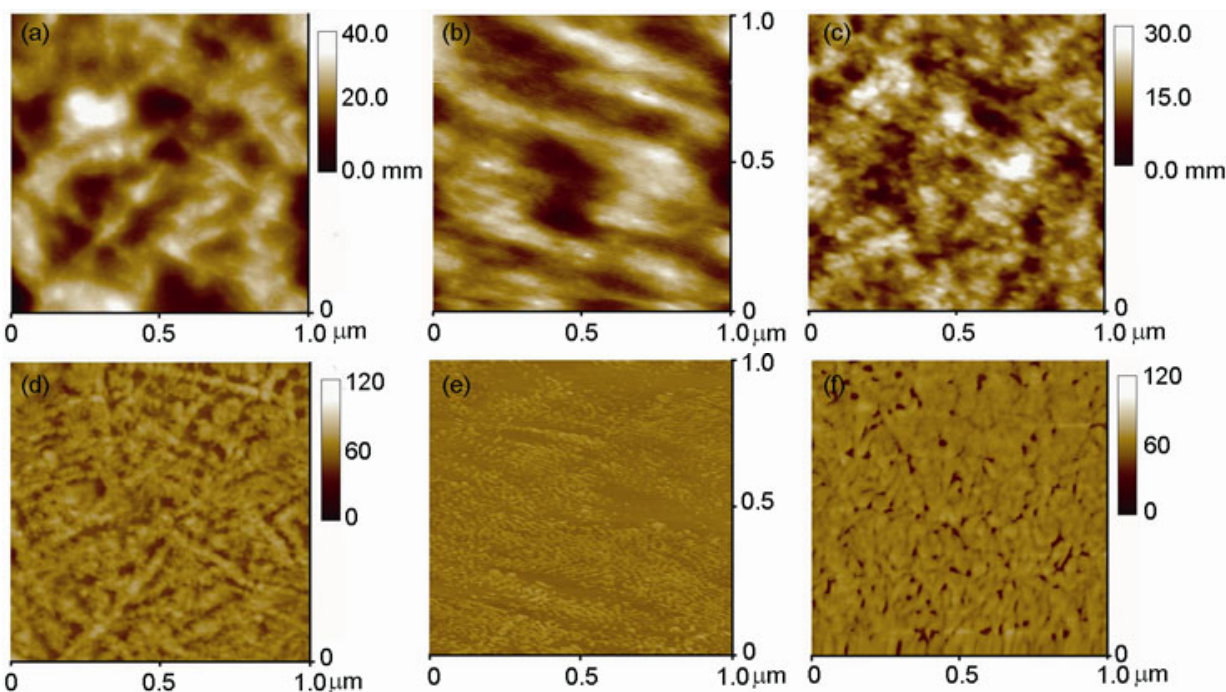

Figure 4 Tapping mode AFM images of P3HT:PCBM blend films: pristine annealed P3HT film ((a), (d)), as-cast P3HT:PCBM blend film ((b), (e)) and annealed P3HT:PCBM blend film ((c), (f)). The above images show height images and below ones show the corresponding phase images, and the scanning scale is $1 \mu \mathrm{m} \times 1 \mu \mathrm{m}$.
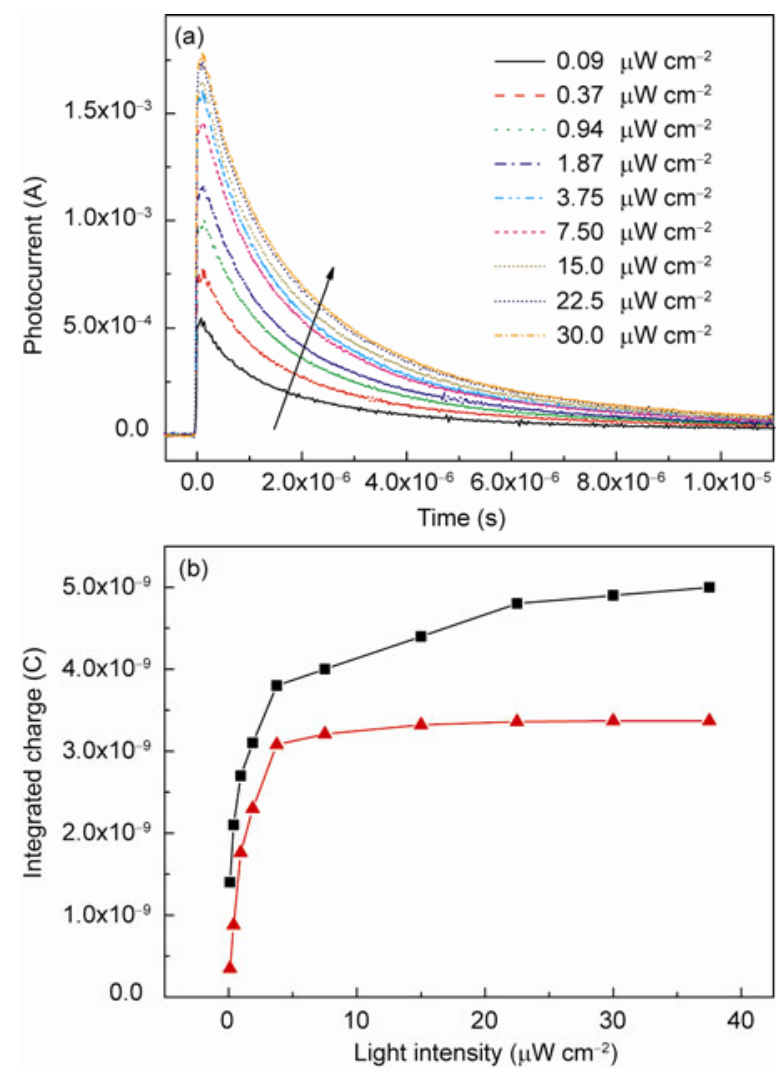

Figure 5 (a) Transient short-circuit photocurrent kinetics of the P3HT: PCBM cell. (b) Light intensity dependence of integrated charge in different P3HT:PCBM solar cells: as-cast device (red line) and annealed device (black line).

below for the extracted charge $Q_{e}[31,32]$.

$$
Q_{e}=\int_{0}^{\infty} j_{e} d t=\frac{e d S}{\beta t_{e}}
$$

where $e$ is the electronic charge, $S$ is the electrode area, $d$ is the thickness of the device, $t_{e}$ is the extracted time, which is approximately equal to $t_{1 / 2}$. Our measured value of $Q_{e}$ is equivalent to the integration of the extracted current against the time. We calculated the bimolecular recombination coefficient $\beta$ of P3HT:PCBM devices with different efficiencies, i.e., $\beta=9.5 \times 10^{-12} \mathrm{~cm}^{3} \mathrm{~s}^{-1}$ for the as-cast device (low efficiency, $\mathrm{PCE}=1.31 \%$ ), and $\beta=3.5 \times 10^{-13} \mathrm{~cm}^{3} \mathrm{~s}^{-1}$ for the annealed device (high efficiency, PCE $=1.97 \%$ ). We attribute this variation to differences in the nanostructure of the active layers of these photovoltaic devices. After annealing treatment, the P3HT:PCBM blend films can form ordered lamellae with better integrating networks and a better phase separation, which result in a better performance of P3HT:PCBM solar cell. The changed nanomorphology also affects the charge transport and recombination dynamics in the bulk heterojunction solar cell [33-35].

For TPV measurement, the device should be connected to a $1 \mathrm{M} \Omega$ input terminal of the digital storage oscilloscope in order to provide open-circuit conditions $(J \sim 0)$ in the device. At this condition, there is almost no current flow through the external circuit, which forces the photogenerated charge carriers to remaine in the bulk of the device. Therefore, the TPV decay measurements can be used to monitor the charge recombination kinetics in polymer solar cells at open circuit [36]. In our experiment, transient photovoltage decay was measured over a range of light intensity (Figure 6(a) and (b)) in different devices of varied conversion efficiencies. As the light intensity increases, the TPV decay dynamics become much faster due to the recombination of the charge carriers. Since the TPV decay can be a measure of polaron population [21], and the TPV decay data can be fitted with a power-law function of $\Delta V \propto$ $t^{-\alpha}$. Such power law kinetics is known to follow one process 

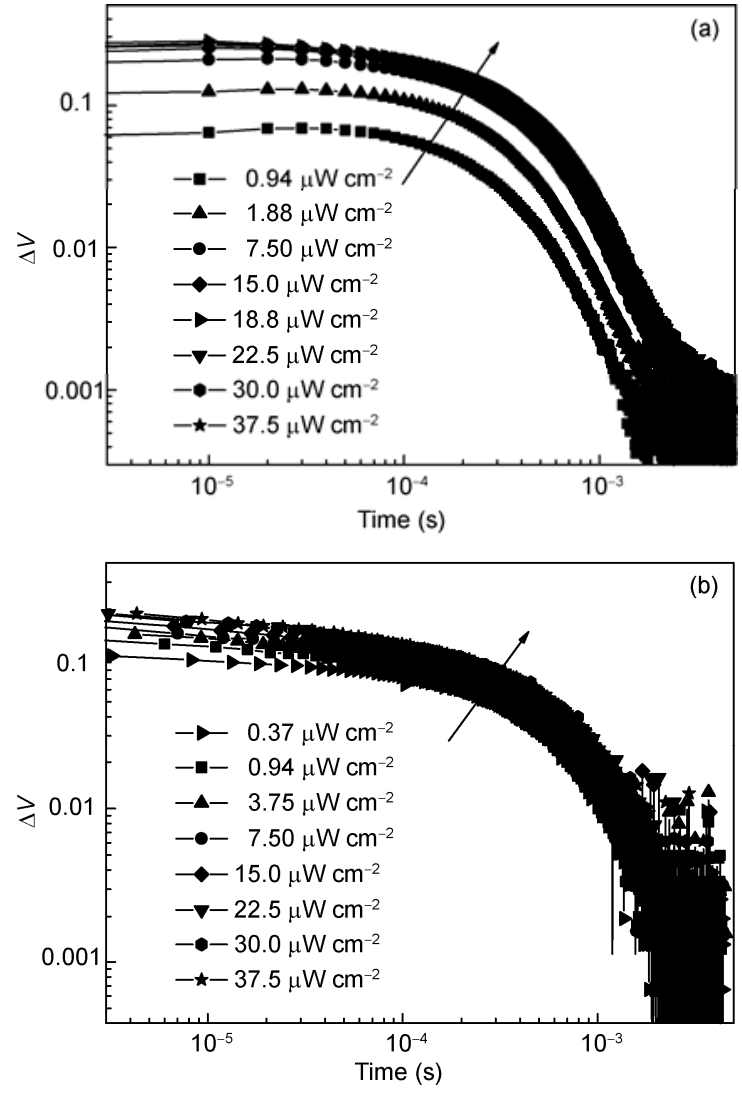

Figure 6 Transient photovoltage decay data of P3HT:PCBM solar cell before (a) and after (b) solvent annealing treatment. The excitation intensity was varied from 0.94 to $37.5 \mu \mathrm{W} \mathrm{cm}$.

which is limited by thermal emission from an exponential density of trap states [36]. The exponent $\alpha$ for high power efficiency device of $1.97 \%$ is 2.6 , the possible mechanism is trap-free bimolecular recombination or multistep tunneling recombination of trapped carriers [36]. For low power efficiency device, the exponent $\alpha$ is 1.76 , which is characteristic of bimolecular recombination of trapped carriers having an exponential tail of polaron trap states (means trap-limited bimolecular recombination) [36-39].

According to the discussion above, the deep trap states are filled by the charge carriers at first, and the the shallower trap states will be filled by the remaining charge carriers. We can conclude that the annealed device with high power efficiency has less trap states, so the charge carriers can be transported in the bulk of the device and collected effectively in the corresponding electrodes. These results suggest that the charge carriers recombination dynamics is strongly dependent on the film nanomorphology, the reduced bimolecular recombination (the value of $\beta$ is small) allows more charge carriers can be collected effectively so that the conversion efficiency is much higher. We can infer that the nanostructure of P3HT:PCBM blend film has a significantly better phase separation after solvent annealing. According to recent studies, it has have shown that there are mainly three phases in P3HT:PCBM blend films, i.e., fibrillar network of P3HT crystals, aggregates of PCBM nanocrystals, and relatively disordered P3HT matrices with PCBM nanocrystals $[40,41]$. The charge carriers transfer freely in fibrillar network of P3HT:PCBM crystals due to the ordered nanostructure of the blend films, which is called trap-free bimolecular recombination. In contrast, the charge carriers transfer in disordered P3HT blend films shows a traplimited bimolecular recombination. The annealed P3HT blend films have more ordered nanostructure, and can improve the charge carriers transport. In conclusion, our results show the correlation between bimolecular recombination coefficient $\beta$, power-law exponent $\alpha$ and nanomorphology of P3HT:PCBM solar cells. This has been discussed above and an increase in charge collection upon solvent annealing treatments due to reduced charge recombination, which is attribute to increased short circuit current and device conversion efficiency.

\section{Conclusion}

In summary, we investigated the transient photocurrent and transient photovoltage decays of different nanostructure P3HT:PCBM blend films before and after solvent annealing treatment. We proposed a possible mechanism for the charge carrier loss in the P3HT:PCBM solar cells. After annealing treatments, P3HT has the required time to form ordered nanostructure, which can provide more percolation pathways for the charge carrier transport and reduce the possibility of recombination.

We are grateful to Professor Leif $H$. Skibsted for reading and revising the manuscript. This work was supported by the National Natural Science Foundation of China (20933010, 21133001 and 21173266), and the National Basic Research Program of China (2009CB20008).

1 Peet J, Heeger A J, Bazan G C. "Plastic"solar cells: Self-assembly of bulk heterojunction nanomaterials by spontaneous phase separation. Acc Chem Res, 2009, 42: 1700-1708

2 Deibel C, Dyakonov V. Polymer-fullerene bulk heterojunction solar cells. Rep Prog Phys, 2010, 73: 096401

3 Scully S R, McGehee M D. Effects of optical interference and energy transfer on exciton diffusion length measurements in organic semiconductors. J Appl Phys, 2006, 100: 034907

4 Shaw P E, Ruseckas A, Samuel Ifor D W. Exciton diffusion measurements in poly(3-hexylthiophene). Adv Mater, 2008, 20: 3516-3520

5 Shaheen S E, Brabec C J, Sariciftci N S, et al. Organic solar cells. Appl Phys Lett, 2001, 78: 841-843

6 Park S H, Roy A, Beaupre S, et al. Organic solar cells, state of the art and future challenges. Nat Photonics, 2009, 3: 297-303

7 Chirvase D, Parisi J, Hummelen J C, et al. Influence of nanomorphology on the photovoltaic action of polymer-fullerene composites. Nanotechnology, 2004, 15: 1317

8 Li G, Yao Y, Yang H, et al. "Solvent annealing" effect in polymer solar cells based on poly(3-hexylthiophene) and methanofullerenes. Adv Funct Mater, 2007, 17: 1636-1644

9 Kim H J, Shin M J, Kim Y K. Distinct annealing temperature in polymer:fullerene:polymer ternary blend solar cells. J Phys Chem C, 
2009, 113: 1620-1623

10 Hoppe H, Niggemann M, Winder C, et al. Nanoscale morphology of conjugated polymer/fullerene-based bulk-heterojunction solar cells. Adv Funct Mater, 2004, 14: 1005-1011

11 Moulé A J, Meerholz K. Controlling morphology in polymerfullerene mixtures. Adv Mater, 2008, 20: 240-245

12 Douhéret $\mathrm{O}$, Swinnen A, Breselge $\mathrm{M}$, et al. High resolution electrical characterisation of organic photovoltaic blends. Microelectron Eng, 2007, 84: 431-436

13 Leever B J, Durstock M F, Irwin M D, et al. Spatially resolved photocurrent mapping of operating organic photovoltaic devices using atomic force photovoltaic microscopy. Appl Phys Lett, 2008, 92: 013302

14 Reid O G, Rayermann G E, Coffey D C, et al. Imaging local trap formation in conjugated polymer solar cells: A comparison of time-resolved electrostatic force microscopy and scanning Kelvin probe imaging. J Phys Chem C, 2010, 114: 20672-20677

15 Coffey David C, Ginger David S. Time-resolved electrostatic force microscopy of polymer solar cells. Nat Mater, 2006, 5: 735-740

16 Sengupta E, Domanski Anna L, Weber Stefan A L, et al. Photoinduced degradation studies of organic solar cell materials using Kelvin probe force and conductive scanning force microscopy. J Phys Chem C, 2011, 115: 19994-20001

17 Juška G, Arlauskas K, Viliūnas M, et al. Extraction current transients: New method of study of charge transport in microcrystalline silicon. Phys Rev Lett, 2000, 84: 4946-4949

18 Mozer A J, Sariciftci N S, Lutsen L, et al. Charge transport and recombination in bulk heterojunction solar cells studied by the phtoinduced charge extraction in linearly increasing voltage technique. Appl Phys Lett, 2005, 86: 112104

19 Montanari I, Nogueira A F, Nelson J, et al. Transient optical studies of charge recombination dynamics in a polymer/fullerene composite at room temperature. Appl Phys Lett, 2002, 81: 3001-3003

20 Nogueira A F, Montanari I, Nelson J, et al. Charge recombination in conjugated polymer/fullerene blended films studied by transient absorption spectroscopy. J Phys Chem B, 2003, 107: 1567-1573

21 Shuttle C G, O'Regan B, Ballantyne A M, et al. Experimental determination of the rate law for charge carrier decay in a polythiophene: Fullerene solar cell. Appl Phys Lett, 2008, 92: 093311

22 Shuttle C G, Maurano A, Hamilton R, et al. Charge extraction analysis of charge carrier densities in a polythiophene/fullerene solar cell: Analysis of the origin of the device dark current. Appl Phys Lett, 2008, 93: 183501

23 Guo J M, Ohkita H, Benten $\mathrm{H}$, et al. Charge generation and recombination dynamics in poly(3-hexylthiophene)/fullerene blend films with different regioregularities and morphologies. J Am Chem Soc, 2010, 132: 6154-6164

24 Marsh R A, Hodgkiss J M, Seifried S A, et al. Effect of annealing on P3HT:PCBM charge transfer and nanoscale morphology probed by ultrafast spectroscopy. Nano Lett, 2010, 10: 923-930
25 Brown P J, Thomas D S, Kohler A, et al. Effect of interchain interactions on the absorption and emission of poly(3-hexylthiophene). Phys Rev B, 2003, 67: 064203

26 Spano F C, Clark J, Silva C, et al. Determining exciton coherence from the photoluminescence spectral line shape in poly(3hexylthiophene) thin films. J Chem Phys, 2009, 130: 074904

27 Spano F C. Modeling disorder in polymer aggregates: The optical spectroscopy of regioregular poly(3-hexylthiophene) thin films. J Chem Phys, 2005, 122: 234701

28 Kim K, Cook S, Tuladhar S M, et al. A strong regioregularity effect in self-organizing conjugated polymer films and high-efficiency polythiophene:fullerene solar cells. Nat Mater, 2006, 5: 197-203

29 Ma W L, Yang C Y, Gong X, et al. Thermally stable, efficient polymer solar cells with nanoscale control of the interpenetrating network morphology. Adv Funct Mater, 2005, 15: 1617-1622

30 Wu C W, Yang H C, Hou W J, et al. Control of the nanoscale crystallinity and phase separation in polymer solar cells. Appl Phys Lett, 2008, 92: 103306

31 Pivrikas A, Juška G, Mozer A $\mathrm{J}$, et al. Bimolecular recombination coefficient as a sensitive testing parameter for low-mobility solar-cell materials. Phy Rev Lett, 2005, 94: 176806

32 Pivrikas A, Sariciftci N S, Juška G, et al. A review of charge transport and recombination in polymer/fullerene organic solar cells. Prog Photovoltaics, 2007, 15: 677-696

33 Riedel I, von Hauff E, Parisi J, et al. Diphenylmethanofullerenes: New and efficient acceptors in bulk-heterojunction solar cells. Adv Funct Mat, 2005, 15: 1979-1987

34 Sánchez-Díaz A, Izquierdo M, Filippone S, et al. The origin of the high voltage in DPM12/P3HT organic solar cells. Adv Funct Mater, 2010, 20: 2695-2700

35 Shoaee S, Eng M P, Espildora E, et al. Influence of nanoscale phase separation on geminate versus bimolecular recombination in P3HT: fullerene blend films. Energy Environ Sci, 2010, 3: 971-976

36 Nelson J. Diffusion-limited recombination in polymer-fullerene blends and its influence on photocurrent collection. Phys Rev B, 2003, 67: 155209

37 Clarke T M, Jamieson F C, Durrant J R. Transient absorption studies of bimolecular recombination dynamics in polythiophene/fullerene blend films. J Phys Chem C, 2009, 113: 20934-20941

38 Montanari I, Nogueira A F, Nelson J, et al. Transient optical studies of charge recombination dynamics in a polymer/fullerene composite at room temperature. Appl Phys Lett, 2002, 81: 3001-3003

39 Nogueira A F, Montanari I, Nelson J, et al. Charge recombination in conjugated polymer/fullerene blended films studied by transient absorption spectroscopy. J Phys Chem B, 2003, 107: 1567-1573

40 Yang X N, Loos J, Veenstra S C, et al. Nanoscale morphology of high-performance polymer solar cells. Nano Lett, 2005, 5: 579-583

41 van Bavel S S, Sourty E, de With G, et al. Three-dimensional nanoscale organization of bulk heterojunction polymer solar cells. Nano Lett, 2009, 9: 507-513

Open Access This article is distributed under the terms of the Creative Commons Attribution License which permits any use, distribution, and reproduction in any medium, provided the original author(s) and source are credited. 